ISSN 0001-6002/2003/45/4/145-153

Acta Médica Costarricense, $(2003$

Colegio de Médicos y Cirujanos

\title{
Alergia al látex: historia, epidemiología, prevención y tratamiento
}

\section{Oscar Porras}

La alergia al látex de hule natural se ha convertido en un problema importante de salud en los trabajadores de la salud y en pacientes con espina bífida e intervenciones quirúrgicas múltiples. Las medidas preventivas permiten reducir la incidencia y son la mejor estrategia para intervenir en este problema de salud pública. Con el objetivo de aumentar el conocimiento sobre el problema de alergia al látex discutimos los aspectos relacionados con historia, epidemiología, diagnóstico, prevención y tratamiento. Se definen los términos sensibilización y alergia y se describen los aspectos químicos del látex de hule natural y de sus proteínas. La definición de grupos de riesgo permite desarrollar una estrategia en las instituciones de salud que disminuye la exposición y como consecuencia las manifestaciones clínicas de los individuos sensibilizados.

Descriptores: látex, alergia, hule natural, guantes quirúrgicos, alergia al látex, alergia ocupacional, hule natural.

Recibido: 9 de setiembre de 2003

Aceptado: 23 de setiembre de 2003

\section{Introducción}

Los casos de sensibilización y alergia al látex han aumentado en todo el mundo desde que se reconoció el problema en $1979^{1-3}$.

Recientemente la opinión pública en Costa Rica se interesó en el tema por la publicidad que se dio a la sentencia No 2003-1432 de la Sala Constitucional, en respuesta al recurso de amparo interpuesto por un funcionario de la CCSS, que condena a la Institución y la obliga a desarrollar una estrategia para el manejo adecuado del problema ${ }^{4}$.

La hipersensibilidad al LHN se ha convertido, en los últimos 20 años, en una de las causas más frecuentes de morbilidad ocupacional de los trabajadores de la salud y de otras ocupaciones donde se utilizan guantes ${ }^{3}$.

Abreviaturas: HNN, Hospital Nacional de Niños "Dr. Carlos Sáenz Herrera"; CCSS, Caja Costarricense de Seguro Social; AL, alergia al látex; HN, hule natural; LHN, látex de hule natural; GL, guantes de látex; IgE, inmunoglobulina E; PT, prueba cutánea para látex ("prick test”); LN, látex natural.

'Servicio de Inmunología y Reumatología Pediátrica

Hospital Nacional de Niños "Dr. Carlos Sáenz Herrera"

Caja Costarricense de Seguro Social

Correspondencia: Dr. Oscar Porras Madrigal (PhD), Servicio de Inmunología y Reumatología Pediátrica, Hospital Nacional de Niños "Dr. Carlos Sáenz Herrera”, Apartado 16541000 San José, Costa Rica.

Facsímil: (506) 2235125. Email: oporras@hnn.sa.cr
La industria de productos basados en $\mathrm{HN}$ utiliza actualmente alrededor de 4.5 millones de toneladas anuales de este material ${ }^{5}$. El LHN es el material que se utiliza en la elaboración de bombas de hule, condones, materiales eléctricos, guantes y otros productos de uso médico. El LN es un polímero que está en contacto con el ser humano en todas sus actividades diarias y con el personal de salud en especial por periodos más prolongados y frecuentes cuando utiliza guantes o materiales médicos descartables ${ }^{1,2}$.

Varias organizaciones, se han interesado en estudiar el tema y en definir políticas y estrategias adecuadas. La Comisión Europea analizó, en su opinión emitida en el año 2000, la sensibilización a proteínas del LHN. En el documento identifican grupos de riesgo, distinguen entre sensibilización y alergia y plantean una posición para abordar el problema dentro de la comunidad ${ }^{6}$.

El problema se origina cuando se genera hipersensibilidad mediada por $\operatorname{IgE}$ contra las proteínas en el látex extraído del árbol de hule Hevea brasiliensis (orden Euphorbiales familia Euphorbiaceae). Los pacientes sensibilizados desarrollan manifestaciones de alergia ante el contacto con látex y cuando se les toca con las manos protegidas por GL ${ }^{7-9}$.

El tema de AL en trabajadores de la salud y en pacientes es controversial en varios de sus aspectos y no hay consenso en relación con el diagnóstico, el tratamiento y la prevención. 
El objetivo de esta revisión es aportar al conocimiento general sobre el LHN y sus problemas de sensibilización, discutir el diagnóstico de sensibilización y alergia y ofrecer recomendaciones para el manejo y prevención del problema en los centros de trabajo, en el personal de salud, en grupos de riesgo y en los pacientes afectados.

\section{Historia}

El uso de látex se documenta en Mesoamérica desde el 1600 $\mathrm{BC}$, en bolas de hule, encontradas en el sitio Manatí en Veracruz, México ${ }^{10}$. Otros artefactos encontrados en Mesoamérica incluyen: agarraderas para herramientas, figuras humanas y moldes de manos humanas. La presencia de canchas para el juego de pelota en la mayoría de los sitios ceremoniales Mayas indica que la habilidad para procesar látex y producir bolas de hule se generó siglos antes de que los Europeos arribaran a Centro América ${ }^{2,10}$. En los primeros artículos de hule elaborados en Mesoamérica, se utilizó material obtenido del árbol Castilla elastica. El hule de C. elastica cuando se seca es quebradizo, por lo que lo mezclaron con el jugo de Ipomoea alba, una enredadera de flores blancas, para mejorar su capacidad de mantener las formas moldeadas y retener elasticidad ${ }^{2}$. Durante la colonia se menciona en varios textos el HN y sus productos en las actividades de la vida diaria de las comunidades indígenas de la época ${ }^{11}$.

Hoy día casi todo el hule natural se obtiene del látex extraído de las plantaciones de $H$. brasiliensis, que crecen en Malasia, Tailandia, India, Centro y Sur América. Otra fuente comercial de látex es el arbusto Guayule (Parthenium argentatum) y de menos uso comercial (producción de goma de mascar) el que se obtiene de árboles de la familia Sapotaceae ${ }^{2}$.

El producto de hule natural que con más frecuencia se asocia con problemas de sensibilización y alergia es el guante de látex para uso médico.

La idea del uso de guantes de hule en procedimientos médicos fue adoptada por los médicos en el periodo 1810-1910. La primera indicación de usar guantes para reducir el riesgo de infección, fue de Adam Elias von Siebold en 1813. En 1843 el descubrimiento de la vulcanización por Charles Goodyear y Nathaniel Hayward en los Estados Unidos y Thomas Hancock en Inglaterra permitió la estabilidad del hule y la producción de guantes. En 1878 se patentaron los primeros guantes de hule para uso quirúrgico. El uso regular de guantes en las actividades médicas se inicia en 1892 con el Dr. Joseph Bloodgood en el Hospital Johns Hopkins ${ }^{2,11}$. Para principios de 1900 los cirujanos en Europa y en los Estados Unidos habían adoptado el uso de los guantes de hule en los actos quirúrgicos y su uso se incrementó a partir de la Segunda Guerra Mundial. Sin embargo los guantes se reservaron para los actos quirúrgicos, hasta su uso generalizado, como barrera de protección en diferentes actividades médicas, como consecuencia de la epidemia de SIDA. El mayor incremento en el uso de los guantes de látex se produjo en respuesta a las recomendaciones para precauciones universales emi- tidas por el CDC en 1987 y $1989^{12,13}$. El número de pares de guantes utilizados aumentó de $12 \times 10^{9}$ en 1987 a más de 200 x $10^{9}$ una década después ${ }^{5}$. El uso de guantes de látex en otras actividades como la manipulación de alimentos se incremento también a partir de $1987^{2}$.

La primera reacción documentada al hule natural se registró en 1927, en 2 pacientes que desarrollaron urticaria y edema laríngeo después de la exposición durante un procedimiento odontológico ${ }^{2,14}$. El segundo reporte tiene que ver con asma y exposición laboral a los vapores producidos por el calentamiento en la cobertura de hule de cables eléctricos. En 1933 los afectados presentaron reacciones de piel al hule de los guantes que utilizaban en sus actividades con compañías de electricidad ${ }^{2}$. El concepto de una reacción alérgica inmediata no se aplicó hasta 1979, en un reporte de reacciones de piel en una mujer atópica al exponerse a un par de guantes de hule, una prueba de parche en piel con parte del guante provocó una reacción local en los siguientes 15 minutos, la reacción alérgica fue comprobada además con una prueba cutánea utilizando un extracto de un guante de hule y un extracto de una hoja de $H$. brasiliensis ${ }^{15,16}$.

\section{Bioquímica}

El LHN químicamente es cis-1,4-poliisopreno, el fluido lechoso que se obtiene principalmente del árbol H. brasiliensis ${ }^{1,5,15}$.

El producto está contenido en células especializadas, los laticíferos. Alrededor de 2000 especies vegetales producen látex, sin embargo más del $99 \%$ del producto comercial proviene de $H$. brasiliensis. El contenido proteico varía entre 1-1,8\% dependiendo de la clona, el clima, el tipo de suelo y la fertilización. Poliisopreno representa el 25-45\% del contenido del LHN. Las proteínas identificadas son alrededor de 200 siendo heveina y hevamina las principales. El LHN también contiene lípidos, carbohidratos y sustancias inorgánicas como potasio, magnesio, calcio, sodio, zinc, manganeso, hierro y cobre. La mayoría de las proteínas del HN se encuentran presentes en los productos terminados de látex ${ }^{17-20}$.

Los guantes son la fuente principal de proteínas de látex en el entorno del ser humano. Los GLcontienen proteínas que fueron sometidas a hidrólisis y desnaturalización durante el proceso de manufactura. Las partículas de látex son insolubles en agua, pero la presencia de amonio que se utiliza para estabilizarlo y preservarlo aumenta su solubilidad. El amonio rompe las organelas presentes en el látex y libera el material soluble. Los fragmentos de las proteínas originan polipéptidos de bajo peso molecular. En el látex amoniado se han detectado cerca de 240 polipéptidos diferentes, con masa molecular entre 5-200 kd, sin embargo solo el $25 \%$ de estos péptidos, con masa molecular entre $5-100 \mathrm{kd}$, muestran ligamiento con $\operatorname{IgE}$ del suero de pacientes con $\mathrm{AL}^{1,5,11,17,21}$.

El principal alergeno en el látex lo constituye el factor de elongación del hule (Hev b). Hay 11 proteínas Hev b (Hev b1 a Hev b11), dentro de estas Hev b5 (18-20 kd) o profilina del 
látex y Hev b6.02 (4,7 kd) heveina. La sensibilización a Hev b5 es frecuente en el grupo de trabajadores de la salud. La concentración de Hev b1 en productos de $\mathrm{HN}$ se reporta entre $18-40 \mu \mathrm{g} / \mathrm{g}$ de $\mathrm{HN}$, lo cual corresponde a $2-4 \%$ de la proteína total extractable de guantes. Hev b6.02 y Hev b5 son responsables de la mayor parte de los alergenos de látex en los guantes de uso médico ${ }^{1,3,11,17-20}$.

Los fabricantes de guantes usan métodos, estándares y tiempos de proceso diversos, lo que produjo, por ejemplo, diferencias de hasta 3000 veces en el contenido de látex en 10 marcas diferentes de guantes estudiadas ${ }^{1,5,17}$.

El almidón de maíz que se utiliza como polvo para lubricar los guantes, actúa como un transportador de alergenos al ligarse a las proteínas de látex. Cuando las partículas se aerolizan durante el uso de los guantes, se produce la exposición al látex de todos los individuos en el área ${ }^{15}$. El polvo de almidón de maíz se adhiere a partículas de látex y actúa como transportador. El talco (silicato de magnesio) es capaz de ligar partículas de látex, sin embargo la unión es irreversible y no se puede liberar el látex al ambiente. Además, el talco, es más pesado y esto hace que sea un mal transportador de aeroalergenos. El cambio de talco por polvo de almidón de maíz en la lubricación de GL, se considera una de las causas del aumento en el número de individuos sensibilizados al $\mathrm{LHN}^{22}$.

Otra vía de exposición es la que ocurre cuando los alergenos del látex entran en contacto con tejidos y mucosas del paciente durante procedimientos quirúrgicos, odontológicos o médicos ${ }^{15}$.

Los productos de $\mathrm{HN}$ pueden fabricarse de látex natural que es una suspensión coloidal concentrada o de hule natural seco que es a base de látex natural coagulado. El hule natural seco es menos alergénico pero no es una alternativa para producir materiales para los individuos con $\mathrm{AL}^{1,3,17,21}$.

El látex que se obtiene del arbusto Guayule no contiene proteínas que produzcan reacciones cruzadas con alergenos del látex de Hevea. Se han demostrado reacciones cruzadas entre proteínas del látex de árboles de los géneros Ficus benjamina, Ficus carica, Ficus elastica, Landolphya kirkil, Sapotaceas y Hevea (Seringa o árbol del Pará) ${ }^{2,11}$. Otras plantas de la familia Euphorbiaceae que pueden estar envueltas en sensibilización son Ricinus communis y Mercurialis anпиа ${ }^{11}$.

Es importante distinguir el $\mathrm{HN}$ de los elastómeros sintéticos, como: el hule butílico (derivado de petróleo), los polímeros de 2-clorobutadieno (Neopreno®) y copolímeros de butadieno y acrilonitrilo. Los productos de hule sintético no representan un riesgo para personas sensibilizadas a las proteínas de $\mathrm{HN}^{21}$.

\section{Reacciones asociadas al látex}

El contacto del ser humano con LHN produce reacciones de diferente tipo, que se explican por mecanismos inmunológi- cos diferentes ${ }^{15}$. El LHN puede actuar como un alergeno de contacto o como un aeroalergeno. Es importante tener claro las diferencias, identificar el origen del problema y responder con soluciones basadas en evidencia. Todas las reacciones se parecen desde el punto de vista clínico ${ }^{15}$.

Un individuo tiene "sensibilización al látex" cuando se demuestra la presencia de anticuerpos tipo IgE contra las proteínas del LHN y no tiene manifestaciones clínicas. Se define como "alergia al látex" cualquier reacción al látex mediada por mecanismos inmunológicos que se acompaña de manifestaciones clínicas. Las reacciones inmunológicas al látex pueden ser de hipersensibilidad Tipo I o Tipo IV 1,10,15,23,24.

Las manifestaciones clínicas pueden ser agudas (hipersensibilidad tipo I) o crónicas (hipersensibilidad tipo IV y dermatitis de contacto) ${ }^{25}$.

Reacciones irritantes de contacto: son las más frecuentes, se desarrollan en periodos cortos (minutos) o largos (horas) después de la exposición a guantes, productos químicos o al látex en su forma natural. Usualmente son benignas, con hiperemia, prurito y ardor en el sitio de contacto $1,5,10,17,24$.

Dermatitis de contacto alérgica o reacción Tipo IV de hipersensibilidad mediada por células: es una reacción de hipersensibilidad retardada como resultado de la generación de Linfocitos $\mathrm{T}$ sensibilizados a los aditivos del látex. Es una respuesta a los oxidantes y aceleradores del hule, como tiouranos, carbamatos y mercapto complejos. Si la exposición es repetida, la reacción se inicia en las siguientes 48-72 horas con eritema, vesículas y descamación. El diagnóstico se realiza aplicando pruebas de parche 1,5,17,25. $^{2}$.

Reacción de hipersensibilidad tipo I, mediada por IgE: esta es la reacción más grave y la que produce mayor morbilidad y mortalidad. Requiere sensibilización y la producción de $\operatorname{IgE}$ específica. Durante la exposición inicial se produce la sensibilización del individuo con la generación de IgE específica contra Hev b. Las proteínas Hev b actúan como antígenos, activan los Linfocitos CD3+4+Th2 y los Linfocitos B, que se diferencian a células plasmáticas y producen $\operatorname{IgE}$ específica anti-Hev b, la cual se liga a receptores en la superficie de mastocitos tisulares y basófilos circulantes. Cuando se produce una segunda exposición, las proteínas Hev b entrecruzan la IgE ligada en la superficie de las células e inducen la degranulación de los mastocitos y basófilos sensibilizados. Los mediadores liberados: histamina, proteasas, metabolitos del ácido araquidónico (leucotrienos y prostaglandinas), conducen a reacciones que fluctúan en intensidad entre urticaria y anafilaxis $1,5,17,24$.

Sindrome frutas-látex: algunas frutas, como banano y aguacate, contienen proteínas que desarrollan reacciones cruzadas con el látex. El látex sin procesar y el de los guantes, el aguacate y los bananos tienen en común un epitopo de $30-\mathrm{kd}^{1,17,18,26}$. También se han encontrado epitopos comunes en antígenos de fresas, cítricos, guabas y peras y se han 
reportado reacciones cruzadas entre látex y mango ${ }^{21}$. Otros alimentos en los que se han documentado reacciones cruzadas con el látex son: kiwi, sandía, melón, papaya, papa, tomate y castañas ${ }^{1,21,23,27}$. La frecuencia de la asociación entre AL y alergia a los alimentos se reporta en $21,1 \%$ (rango 21-58\%) y la de alergia a las frutas con AL en $86,0 \%$ (4\% en los controles) ${ }^{1,15,17,27}$. Los pacientes alérgicos a frutas tienen un riesgo de reaccionar con látex del $11 \%$, pero los pacientes con ALtienen un riesgo de reaccionar contra las frutas de $35 \%{ }^{26}$. Las proteínas asociadas con quitinasa se consideran la principal fuente de reacciones cruzadas entre látex y frutas ${ }^{17,28,29}$.

Las proteínas de látex se absorben lentamente cuando la exposición es por vía aérea, y los síntomas se desarrollan alrededor de 30 minutos después. Las reacciones como urticaria, rinitis y conjuntivitis se asocian con contacto directo sobre la piel o con partículas disueltas en el aire. El contacto entre partículas de almidón de maíz con proteínas de látex que se genera cuando se usan guantes con polvo lubricante, produce broncoconstricción, rinitis y conjuntivitis.

La sensibilización y los síntomas respiratorios se generan con frecuencia cuando la concentración de alergenos de látex en el aire es mayor de $0,6 \mathrm{ng} / \mathrm{m}^{3}$ y se asocian con el tiempo de permanencia en la sala de operaciones, el número de guantes usados y el uso de guantes con alto contenido de alergenos ${ }^{30,31}$.

Las reacciones graves usualmente ocurren en el corto plazo después del contacto con mucosas y piel: enrojecimiento, vasodilatación, broncoespasmo y aumento de la permeabilidad vascular con edema y colapso cardiovascular ${ }^{23}$.

En un estudio con anestesistas se identificó un 12,5\% de sensibilizados, $2,4 \%$ con síntomas de alergia y el 10,1\% restante no mostró síntomas incluso después de estudios de provocación en piel y vía respiratoria ${ }^{32}$.

Debe realizarse un adecuado diagnóstico diferencial con otras posibles causas de manifestaciones alérgicas y de anafilaxia dentro del entorno de la sala de operaciones o de las actividades que realiza el paciente o el personal de salud sensibilizado ${ }^{1,3,5,17}$.

\section{Diagnóstico}

Se realiza con una historia y examen físico en relación con las manifestaciones clínicas asociadas a la exposición al látex y un estudio de laboratorio positivo ${ }^{1}$.

El diagnóstico se debe plantear, cuando después de la exposición al látex se presentan síntomas como enrojecimiento de manos, rinitis, conjuntivitis, dificultad respiratoria, tos, sibilancias, anafilaxia o estado de choque. Ante la presencia de síntomas se requiere una evaluación que permita desarrollar una estrategia de prevención y tratamiento que evite una reacción alérgica grave provocada por la exposición repetida al LHN ${ }^{5}$.
Un diagnóstico durante la reacción aguda, es posible en países con la tecnología para determinar triptasas en suero. La $ß$-triptasa es una proteasa neutra que se almacena en los gránulos secretores de los mastocitos, su incremento sérico indica la activación de los mastocitos con liberación de mediadores, el pico se alcanza a los 30 minutos y luego disminuye gradualmente, su vida media es de 2 horas. Un nivel de triptasas $>13 \mathrm{ng} / \mathrm{mL}$ o de $\beta$-triptasa $>1 \mathrm{ng} / \mathrm{mL}$ es específico para anafilaxis ${ }^{1,33,34}$

Los estudios serológicos permiten la cuantificación de anticuerpos específicos tipo IgE contra látex, se pueden realizar cuando hay manifestaciones de piel, durante tratamiento con antihistamínicos y en periodos cercanos a episodios de anafilaxis. En general la técnica empleada es RAST ("RadioAllergoSorbent Test"), que tiene alta especificidad (80-70\%) pero baja sensibilidad (50-90\%). La tasa de falsos positivos se asocia principalmente con la presencia de anticuerpos contra carbohidratos que producen reacción cruzada con los anticuerpos IgE contra látex. La tasa de falsos negativos es de $25 \%$ y requieren de interpretación cuidadosa en presencia de una historia sugestiva de AL. No se recomienda tamizar pacientes antes de procedimientos quirúrgicos sino hay una historia sugestiva de sensibilización ${ }^{1,3,5,17,35}$.

Las pruebas cutáneas se deben realizar 4-6 semanas después del episodio de anafilaxia, por la depleción de mediadores de los mastocitos. Se utilizan extractos de látex con amoniaco, látex sin amoniaco y extractos de guantes de hule. Todos los extractos resultan equivalentes en sensibilidad y especificidad si se estandarizan por el contenido de proteína ${ }^{1,8,17}$.

El algoritmo para el diagnóstico de hipersensibilidad al látex, es la etapa inicial del manejo. Si identificamos una reacción de hipersensibilidad tipo IV por la naturaleza tardía de la clínica y reacciones en el lugar de contacto, se debe proceder a una prueba de parche, para confirmar la presencia de linfocitos Tactivados con especificidad para los químicos asociados al hule ${ }^{21}$. Si la reacción es de hipersensibilidad tipo I, con síntomas oculares, respiratorios, de piel y sistémicos de inició rápido después de la exposición, debemos proceder a la determinación en suero de IgE específica o una prueba cutánea ("Prick Test") que demuestre la sensibilización ${ }^{20,21,35,36}$.

La prueba definitiva de AL es la provocación con látex, sin embargo implica el riesgo de desencadenar manifestaciones clínicas graves y no hay métodos estandarizados. Su indicación principal es en los casos en que hay discordancia entre la historia clínica y las pruebas in vitro o bien si hay ausencia de síntomas en una paciente con serología o prueba de piel positiva. Pueden realizarse con técnicas de contacto o de inhalación. Un ejemplo es la prueba en la cual se usa un GL durante 15 minutos y se miden las reacciones comparando con un guante de vinilo. La prueba de provocación mas utilizada es la de uso de guante o de dedo de guante, en casos seleccionados se realiza una prueba de inhalación de polvo de guante g, $^{3,35}$. 


\section{Epidemiología}

Durante los últimos 20 años, el látex se ha posicionado como la segunda causa de anafilaxis en salas de operaciones ( $16,6 \%$ de los casos). Sin embargo la incidencia ha descendido en respuesta a la identificación de pacientes de riesgo, mejoras en las determinaciones de laboratorio, introducción de medidas de prevención y reducción de látex en muchos de los productos médicos.

Apartir de 1980 se identificó un aumento súbito en el número de casos de AL. Varios factores explican este fenómeno:

a) El descubrimiento de la existencia de la AL, indujo criterios de diagnóstico y como consecuencia un aumento en los casos reportados.

b) El uso generalizado de guantes en las actividades del personal de salud, como consecuencia de las medidas de bioseguridad universal implantadas ante la epidemia del SIDA.

c) El aumento en la demanda de guantes para uso médico produjo una reducción en la calidad de los productos.

d) El contenido de alergenos en los guantes aumento a partir de 1980, debido a la transferencia de la manufactura de los países industriales a los países productores de látex, este cambio produjo cambios en un proceso que tardaba meses, durante los cuales el látex estaba mezclado con amonio, a uno en el país productor de 4 a 6 semanas. El contacto prolongado con amonio produce hidrólisis de las proteínas de látex dejando pocas proteínas intactas y produciendo un material menos alergénico para iniciar el proceso de manufactura.

e) La selección de especies de alta producción de látex y el uso de agentes químicos que se aplican a los árboles para aumentar la producción. Árboles de alta producción producen látex con mayor contenido de proteínas. Los agroquímicos utilizados usualmente son derivados de etileno, que se aplica también en las frutas, la reacción con las proteínas puede inducir reacciones cruzadas entre látex y frutas, incrementando las posibilidades de sensibilización ${ }^{2}$.

La prevalencia de sensibilización al látex es $<1 \%$ en la población normal sin atopia, pero en el personal de salud fluctúa entre 3 y $12 \%{ }^{1,17,37}$. Utilizando el PT, la prevalencia en población no seleccionada de positividad es de $0,12 \%$. Si se utiliza la determinación en suero de $\mathrm{IgE}$ específica contra látex 4-6,4\% de los individuos son positivos ${ }^{37}$. La incidencia de sensibilización al látex (medida como nivel de IgE específica) en pacientes quirúrgicos ambulatorios es de $6,7 \%{ }^{1}$. La prevalencia de AL en la población general es de $0,7-.11 \%{ }^{37}$.

En un grupo de individuos no sensibilizados que inician su actividad en un área de salud se demostró una incidencia acu- mulada para sensibilización cutánea de $6,4 \%$, para rinoconjuntivitis de $1,8 \%$ y para asma ocupacional de $4,5 \%{ }^{38}$.

Se requieren sin embargo más estudios epidemiológicos para describir adecuadamente los grupos de riesgo, las diferencias entre trabajadores de la salud y la población general y la relación con atopia y otras variables demográficas ${ }^{39}$.

Grupos de riesgo: los grupos de alto riesgo para desarrollar AL incluyen: trabajadores de la salud o con exposición ocupacional al látex (policía, estilistas, manipuladores de alimentos), individuos con historia de atopia y pacientes con espina bífida y anomalías genitourinarias que requieren múltiples intervenciones quirúrgicas, ${ }^{1,3,17,40}$.

Los pacientes con espina bífida, aún sin múltiples cirugías, tienen un riesgo aumentado de AL. La presencia de espina bífida, atopia y el número de intervenciones quirúrgicas son factores de riesgo independientes. Hev b1 es el principal alergeno en este grupo. La manifestación más frecuente de AL asociada a espina bífida es urticaria ${ }^{1,41-43}$. Obojski ${ }^{43}$ en su grupo de estudio reportó prevalencias de $32,4 \%$ y $18,8 \%$ para sensibilización y AL respectivamente en niños (as) con espina bífida. Se ha reportado diferencias entre comunidades en países con poca intervención quirúrgica y países con facilidades libres de látex, sin embargo la incidencia de sensibilización y síntomas ha aumentado ${ }^{44}$. En niños con espina bífida y enfermedades congénitas que requieren cirugía múltiple la frecuencia se reporta entre $23-70 \% \%^{3,5,42,43,44}$.

La exposición ocupacional al látex es frecuente en personal de salud que se cambia guantes varias veces durante sus turnos de trabajo. Las enfermeras de unidades de cuidado intensivo, en los Estados Unidos, se cambian guantes 50-100 veces al día y el personal de anestesia 15-20 veces al día ${ }^{1}$.

Los trabajadores de la salud son el principal grupo de riesgo, por exposición constante a productos con látex, la frecuencia de sensibilización en la población hospitalaria fluctúa entre 2,8-17\%. En otras ocupaciones con exposición al látex la frecuencia varia de $5-11 \%$. Alergia en trabajadores de la salud con AL es 2,2-4,2 veces más frecuente que en los trabajadores control, en adultos con AL se reporta una prevalencia de hasta $82 \%{ }^{3}$. Un trabajador de la salud que es atópico tiene un riesgo aumentado de sensibilización y AL.

Los anestesistas tienen un $12,5 \%$ y $2,4 \%$ de prevalencia de sensibilización y alergia al látex respectivamente ${ }^{32}$. Los anestesistas de adultos se cambian los guantes más veces que los de niños y tienen mayor sensibilidad al látex con estimados de afectados de $24 \%{ }^{1,5,32}$.

No hay riesgo asociado con edad, sexo o raza. Exposición es el principal factor asociado con AL, además se identifica una asociación con el uso de guantes con polvo y con alto contenido proteico ${ }^{32}$. 


\section{Prevención y manejo}

La prevención es la intervención más importante en el manejo de la sensibilización al látex. Los pacientes deben recibir educación sobre el problema y las alternativas para evitar la exposición y las reacciones alérgicas 1,15, 45,46,47. La estrategia más efectiva de manejo es evitar la exposición y no la desensibilización ${ }^{15}$.

Los individuos sensibilizados deben tener información sobre productos y ambientes que pueden inducir las manifestaciones clínicas.

El personal y los pacientes sensibilizados al látex deben portar una identificación sobre su condición para situaciones de emergencia y llevar consigo inyectores automáticos de adrenalina tipo Epi-pen ${ }^{\circledR}{ }^{45}$.

Las pruebas cutáneas para látex se mantienen positivas 2 años después de que el paciente inicia las actividades para evitar la exposición al látex, pero los anticuerpos tipo IgE específicos disminuyen, al igual que los síntomas de alergia en respuesta a la intervención.

Se recomienda que los procedimientos quirúrgicos en pacientes con AL se realicen de primeros en la mañana cuando los niveles de aeroalergenos de látex son los menores, lavar las manos para eliminar trazas de polvo o látex, usar un área quirúrgica y materiales libre de látex, y con facilidades de resucitación. Es preferible usar jeringas de vidrio o contar con émbolos sin látex en pacientes con historia de anafilaxia.

El uso de guantes de vinil es una alternativa, sin embargo tienen condiciones de barrera y durabilidad menores que los de LHN ${ }^{1}$.

Inmunoterapia: la alergia mediada por IgE al LHN, se ha convertido en un problema importante de salud, especialmente en el personal de salud, en individuos de riesgo, un tratamiento curativo induciendo tolerancia inmunológica en pacientes sensibilizados es aún experimental y no está disponible en todos los casos. Apesar de que las medidas de prevención que evitan la sensibilización son la estrategia más efectiva, se debe tener presente que la inmunoterapia con desensibilización en las manos de expertos y con las medidas adecuadas para manejar las complicaciones es un tratamiento que ofrece una alternativa en casos seleccionados en los que la reducción de la exposición no es suficiente para manejar las manifestaciones clínicas ${ }^{46}$. En el manejo del paciente con ALse ha utilizado inmunoterapia con desensibilización usando extractos de las proteínas de látex ${ }^{20,48}$. Sin embargo puede inducir reacciones alérgicas sistémicas y requiere más estudios que analicen el efecto a largo plazo y sobre las reacciones cruzadas con frutas ${ }^{49}$.

Otra técnica de desensibilización son los protocolos de exposición. Se describe el uso de GL en una mano del paciente sensibilizado por periodos de tiempo iniciales de 10 segundos
/ día hasta llegar a una exposición de 1 hora en ambas manos dos veces al día al final de un año de tratamiento ${ }^{50}$.

Otra alternativa es el uso de proteínas modificadas (Hev b5, Hev b6.02), que resultan en una actividad ligadora de $\operatorname{IgE}$ menor. Estas proteínas van a inducir poco ligamiento a $\operatorname{IgE}$ en los mastocitos y pocas reacciones durante la exposición lo que las convierte en mejores proteínas para inmunoterapia y más seguras y eficaces en la desensibilización ${ }^{51,18}$.

Se han generado anticuerpos monoclonales humanizados anti-IgE que en los pacientes alérgicos tratados han producido reducción de los síntomas y del uso de medicamentos, lo cual prevé una opción en el manejo la $\mathrm{AL}^{52}$.

Modificaciones del ambiente de trabajo: el uso de GLcon bajo contenido proteico extractable o libres de látex es una medida preventiva efectiva ${ }^{8,953}$. Es necesario generar una estrategia en cada institución de salud para manejar efectivamente el problema, incluyendo reducción de la exposición, manejo de los afectados, educación y métodos adecuados de diagnóstico ${ }^{53-55}$.

El guante ideal es aquel con $<1 \mu \mathrm{gm} / \mathrm{gm}$ de alergeno por la prueba de ELISA-látex para proteínas antigénicas (LEAP) y de 1-14 unidades/mL de alergenos en la prueba de RAST de inhibición y sin polvo lubricante de almidón de maíz. Se debe insistir en que los guantes al menos cumplan con $<10$ LEAP o $<100$ RAST ${ }^{15}$.

Cuando se usan guantes con polvo la exposición fluctúa entre $5-616 \mathrm{ng} / \mathrm{m}^{3}$, si se utilizan guantes sin polvo en la mayoría de los casos la exposición es $<0,1 \mathrm{ng} / \mathrm{m}^{355}$.

El costo de utilizar la estrategia de una: "institución libre de látex", en un centro de salud es menor que el costo de responder a la discapacidad que produce la AL, aún cuando el nuevo de discapacitados sea $<2 \%{ }^{57}$.

La estrategia para manejar el problema de sensibilización al látex en una institución de salud, debe tomar en cuenta el abordaje del personal y de los pacientes sensibilizados y las alternativas para reducir la exposición a los alergenos del LHN ${ }^{57,58}$.

La intervención para reducir la exposición debe tomar en cuenta el uso de productos con bajo contenido de proteínas extractables de látex, y dentro de este grupo de materiales incluir guantes sin polvo lubricante de almidón de maíz. Cuando se utilizan guantes con polvo lubricante la concentración ambiental de aeroalergenos de látex fluctúa dependiendo del estudio y de la técnica de medición entre $0,9-616 \mathrm{ng} / \mathrm{m}^{3}$, con guantes sin polvo lubricante los valores se reportan entre 0,0 $15 \mathrm{ng} / \mathrm{m}^{3}$, valores muy cercanos a las concentraciones de 0,3 $1,8 \mathrm{ng} / \mathrm{m}^{3}$ que se detectan en el aire de ambientes con poco uso de GL ${ }^{55.59-62}$. La dosis total de aeroalergenos de látex que induce una crisis asmática durante una provocación ambiental se ve influida por la duración de la exposición y varia mucho individualmente ${ }^{63}$. 
La medición de la cantidad total de proteína en el GL es el mejor método para determinar la capacidad alergénica de los $\mathrm{GL}^{30,47}$.

Es evidente que el uso de guantes sin polvo lubricante de almidón de maíz y con bajo contenido de proteínas extractables debe ser un criterio para la adquisición de este tipo de materiales dentro de una estrategia institucional para disminuir la exposición al látex.

El etiquetado de los materiales y suministros que son productos de LHN es otra intervención que evita al individuo sensibilizado la exposición al LHN.

Se debe establecer prevención primaria sobre las poblaciones identificadas como de riesgo: como por ejemplo los protocolos en niños con espina bífida para evitar el contacto con látex desde la primera cirugía. Con este tipo de estrategia se reportan reducciones del número de niños (as) sensibilizados (42\% sin intervención vs 7\% con intervención) ${ }^{64,65}$. Otros grupos de riesgo en niños en los que esta intervención se prevé efectiva incluyen pacientes con ventilación asistida domiciliar (66) y con intervenciones quirúrgicas frecuentes por atresia de esófago ${ }^{67}$ y malformaciones del tracto urinario, genital y óseo.

Una sustitución completa de los materiales de látex por alternativas que mantengan protección no es posible en la actualidad. Virus pequeños penetran menos el látex que el polietileno y el vinilo ${ }^{68,69}$. Es difícil sustituirlo con un material que tenga las mismas características de comodidad, elasticidad, preservación del tacto y duración. Además las alternativas, los copolímeros sintéticos (poli isopreno y neopreno), son de mucho más alto costo.

Debemos utilizar entonces GL de alta calidad poco sensibilizantes en las tareas en que son insustituibles y guantes de otros materiales cuando la actividad lo permita.

Se deben planear con cuidado las estrategias para proveer ambientes libres de látex para procedimientos quirúrgicos en pacientes con AL bien diagnosticada ${ }^{21,69}$.

Es importante tomar en cuenta que existen dificultades en establecer alergia ocupacional al látex en pacientes con historia de alergia ${ }^{70}$.

A nivel institucional hay varias alternativas ${ }^{71}$ :

a) No usar productos que contengan LHN

b) Reducir la exposición a proteínas LHN

c) Crear zonas libres de látex, tomando en cuenta que nos se pueden garantizar por tratarse de una proteína transportable por aire y que se puede llevar en la ropa, pelo y documentos. Pero si es posible aplicar esta opción en una área bien aislada de la institución.
Se debe distinguir entre los términos "libre de látex" que indica un producto no manufacturado con LHN y "de uso seguro", el producto de LHN controlado bajo normas y estándares que no produce riesgo para pacientes alérgicos por contacto directo o inhalación.

Un ambiente seguro para látex es el que reduce el riesgo de desarrollar sensibilización por medio de una eliminación significativa de la exposición al LHN ${ }^{15,72}$.

Para optimizar la prevención primaria en los lugares de trabajo, se deben fijar límites de exposición ocupacional para alergenos, basados en parámetros de salud ${ }^{73}$.

\section{Abstract}

IgE-mediated allergy to natural rubber latex has become a significant health problem among health care workers, patients with spina bifida or with a history of multiple surgical interventions. Curative treatment using immunotherapy, is not the most effective approach. Preventive measures reduce latex allergy incidence and are a better strategy for this public health problem. In this article, we discuss history, epidemiology, diagnosis, prevention and treatment of latex allergy. The differences between sensitization and allergy and the chemical aspects of natural rubber and its proteins are analysed. The definition of a risk group allows the development of an strategy aimed to decrease exposure and clinical manifestations among sensitized patients.

\section{Referencias}

1. Hepner DL, Castells MC. Latex allergy: an update. Anesth Analg 2003; 96: 1219-1229.

2. Ownby DR. A history of latex allergy. J Allergy Clin Immunol 2002; 110: S27-32.

3. Alenius H, Turjanmaa K, Palosuo T. Natural rubber latex allergy. Occup Environ Med 2002; 59: 419-424.

4. Sala Constitucional de la Corte Suprema de Justicia. Sentencia 20031432 (6 de Mayo de 2003).

5. Tesiorowski CC. Latex allergies in the health care worker. J Perianesth Nurs 2003; 18: 18-31.

6. Scientific Committee on Medicinal Products and Medical Devices. Opinion on natural rubber latex allergy. European Commission, Health and Consumer Protection Directorate-General, Directorate B - Scientific Health Opinions (27 de Junio de 2000) 2000: 1-35.

7. Turjanmaa K, Reunala T. Condoms as a source of latex allergen and cause of contact urticaria. Contact Dermatitis 1989; 83:1135-1137

8. Turjanmaa K. Diagnosis of latex allergy.Allergy 2001; 56: 810-812.

9. Quirce S, Olaguíbel JM, Alvarez MJ, Tabar AI. El látex. Un importante aeroalergeno implicado en el asma ocupacional. An sis sanit Navar 2003; 26: S81-95.

10. Hosler D, Burkett SL, Tarkanian MJ. Prehistoric polymers: rubber processing in ancient Mesoamerica. Science 1999; 284: 1988-1991.

11. Quirce Gancedo S, Conde-Salazar Gómez L. Obtención y procesamiento del caucho natural. En: Blanco Coverra C, Quirce Gancedo S (ed). Alergia al látex 2002. Sociedad Española de Alergia e Immunología Clínica. Barcelona: MRAEditions; 2002: 13-20.

12. Centers for Disease Control and Prevention. Recommendations for prevention of HIVtransmission in health-care settings. MMWR 1987; 36: $35-185$. 
13. Centers for Disease Control and Prevention. Guidelines for prevention of transmission of human immunodeficiency virus and hepatitis B virus to health-care and public safety workers. MMWR 1989; 38: 1-37.

14. Fuchs T. Latex allergy. J Allergy Clin Immunol 1994; 93: 951-952.

15. Zucker-Pinchoff B. Latex allergy. Mt Sinai J Med 2002; 69: 88-95.

16. Nutter AF. Contact urticaria to rubber. Br J Dermatol 1979; 101: $597-$ 598.

17. Kurup VP, Fink JN. The spectrum of immunologic sensitization in latex allergy.Allergy 2001; 56: 2-12.

18. Karisola P,Alenius H, Mikkola J, Kalkkinen J, Kalkkinen N, Helin J, et al. The major conformational IgE-binding epitopes of hevein (hev b6.02) are identified by a novel chimera-based allergen epitope mapping strategy. J Biol Chem 2002; 277: 22656-22661.

19. Barber Hernández D, Lombardero Vega M. Composición alergénica del latex. En: Blanco Coverra C, Quirce Gancedo S (ed). Alergia al látex 2002. Sociedad Española de Alergia e Immunología Clínica. Barcelona: MRAEditions; 2002: 29-43

20. Sánchez Palacios A. Alergia al látex. Diagnóstico y aspectos terapéuticos. Allergol et Immunopathol 2001; 29: 212-221.

21. Ahmed DD, Sobcsak SC, Yunginger JW. Occupational allergies caused by latex. Immunol Allergy Clin North Am 2003; 23: 205-219.

22. Lundberg M, Wrangsjö K, Johansson SGO. Latex allergy from glove powder-an unintended risk with the switch from talc to cornstarch. Allergy 1997; 52: 1222-1228.

23. Anda M, Gómez B, Lasa E, Arroabarren E, Garrido S, Echechipía S. Alergia al látex. Manifestaciones clínicas en la población general y reactividad cruzada con alimentos. An sis sanit Navar 2003; 26: S7580 .

24. Turjanmaa K, Alenius H, Reunala T, Palosuo T. Recent developments in latex allergy. Curr Opin Allergy Clin Immunol 2002; 2: 407-412.

25. Blanco Guerra C, Almeida Quintana L, García Dumpiérrez A. Manifestaciones clínicas de la alergia al látex. En: Blanco Coverra C, Quirce Gancedo S (ed). Alergia al látex 2002. Sociedad Española de Alergia e Immunología Clínica. Barcelona: MRAEditions; 2002: 47-62.

26. Lavaud F, Prevost A, Cossart C, Guerin L, Bernard J, Kochman S. Allergy to latex, avocado pear, and banana: evidence for a $30 \mathrm{kd}$ antigen in immunoblotting. J Allergy Clin Immunol 1995; 95:557-564.

27. Blanco C. Latex-fruit syndrome. Curr Allergy Asthma Rep 2003; 3: 4753.

28. Sicherer SH. Clinical implications of cross-reactive food allergens. J Allergy Clin Immunol 2001; 108: 881-890.

29. Aalberse RC, Akkerdaas JH, van Ree R. Cross-reactivity of IgE antibodies to allergens. Allergy 2001; 56: 478-490.

30. Baur X, Chen Z, Allmers H. Can a threshold limit value for natural rubber latex airborne allergens be defined? J Allergy Clin Immunol 1998; 101:24-27.

31. Heilman DK, Jones RT, Swanson MC, Yunginger JW. A prospective, controlled study showing that rubber gloves are the major contributor to latex aeroallergen levels in the operating room. J Allergy Clin Immunol 1996; 98:325-330.

32. Brown RH, Schauble JF, Hamilton RG. Prevalence of latex allergy among anesthesiologists: identification of sensitized but asymptomatic individuals. Anesthesiology 1998; 89: 292-299.

33. Schwartz LB, Bradford TR, Rouse C, Irani AM, Rasp G, Van der Zwan JK, et al. Development of a new, more sensitive immunoassay for human tryptase: use in systemic anaphylaxis. J Clin Immunol 1994; 14 : 190-204.

34. Castells MC, Irani AM, Schwartz LB. Evaluation of human peripheral blood leukocytes for mast cell tryptase. J Immunol 1987; 138: 21842189.

35. Blanco Guerra C, García Dumpiérrez A, Almeida Quintana L. Utilidad y limitaciones de las técnicas diagnósticas. En: Blanco Coverra C, Quirce Gancedo S (ed). Alergia al látex 2002. Sociedad Española de
Alergia e Immunología Clínica. Barcelona: MRAEditions; 2002: 101119.

36. Hamilton RG, Peterson EL, Ownby DR. Clinical and laboratory-based methods in the diagnosis of natural rubber latex allergy. J Allergy Clin Immunol 2002; 110: S47-56.

37. Liss GM, Sussman GL. Latex sensitization: occupational versus general population prevalence rates. Am J Ind Med 1999; 35: 196-200.

38. Archambault S, Malo JL, Infante-Rivard C, Ghezzo H, Gautrin D. Incidence of sensitization, symptoms, and probable occupational rhinoconjunctivitis and asthma in apprentices starting exposure to latex. J Allergy Clin Immunol 2001; 107: 921-923.

39. Garabrant DH, Schweitzer S. Epidemiology of latex sensitization and allergies in health care workers. J Allergy Clin Immunol 2002; 110: S82-95.

40. Carrillo Diaz T, Figueroa Rivero J, Navarro Déniz L, Ortega Rodríguez N. Epidemiología y factores de riesgo de la alergía al látex. En: Blanco Coverra C, Quirce Gancedo S (ed). Alergia al látex 2002. Sociedad Española de Alergia e Immunología Clínica. Barcelona: MRAEditions; 2002: 85-97.

41. Hochleitner B-W, Menardi G, Haussler B, Ulmer H, Kofler H, Reider N. Spina bifida as an independent risk factor for sensitization to latex. J Urol 2001; 166: 2370-2374.

42. Obojski A, Chodorski J, Barg W, M?drala W, Fal AM, Ma?olepszy J. Latex allergy and sensitization in children with spina bifida. Pediatr Neurosurg 2002; 37: 262-266.

43. Nieto Garcia A, Mazón Ramos A, Pamies Espinoza R, Díaz Ruiz J. Espina bífida y alergia al látex. En: Blanco Coverra C, Quirce Gancedo S (ed). Alergia al látex 2002. Sociedad Española de Alergia e Immunología Clínica. Barcelona: MRAEditions; 2002: 149-174.

44. Randolph C. Latex allergy in pediatrics. Curr Probl Pediatr 2001; 31: 135-153.

45. Muller BA. Minimizing latex exposure and allergy. How to avoid or reduce sensitization in the healthcare setting. Postgrad Med 2003; 113: 91-97.

46. Brehler R, Kütting B. Natural rubber latex allergy: a problem of interdisciplinary concern in medicine. Arch Intern Med 2001; 161: 1057-1064.

47. Baur X, Chen Z, Raulf-Heimsoth M, Degens P. Protein and allergen content of various natural latex articles. Allergy 1997; 52: 661-664.

48. Tabar AI, Gómez B, Arroabarren E, Rodríguez M Lázaro I, Anda M. Perspectivas de tratamiento de la alergia al látex: inmunoterapia. An sis sanit Navar 2003; 26: S97-102.

49. Sastre Domínguez J. Inmunoterapía con látex. En: Blanco Coverra C, Quirce Gancedo S (ed). Alergia al látex 2002. Sociedad Española de Alergia e Immunología Clínica. Barcelona: MRAEditions; 2002: 247 252.

50. Patriarca G, Nucera E, Buonomo A, Del Ninno M, Roncallo C, Pollastrini $\mathrm{E}$, et al. Latex allergy desensitization by exposure protocol: five case reports. Anesth Analg 2002; 94: 754-758. Patriarca G, Nucera E, Pollastrini E, Roncallo C, Buonomo A, Bartolozzi F, et al. Sublingual desensitization: a new approach to latex allergy problem. Anesth Analg 2002; 95: 956-960. Nucera E, Schiavino D, Pollastrini E, Roncallo C, de Pacuale T, Buonomo A, et al. Desensitization to latex by percutaneous route. J Investig Allergol Clin Immunol 2002; 12: 134-135.

51. Beezhold DH, Hickey VL, Sussman GL. Mutational analysis of the IgE epitopes in the latex allergen Hev b 5. J Allergy Clin Immunol 2001; 107: 1069-1076.

52. Milgrom H, Fick RB Jr, Su JQ, Reimann JD, Bush RK, Wathous ML, Metzger WJ. Treatment of allergic asthma with monoclonal anti-IgE antibody. rhuMAb-E25 study group. N Engl J Med 1999; 341: 19661973.

53. Turjanmaa K, Kanto M, Kautiainen H, Reunala T, Palosuo T. Longterm outcome of 160 adult patients with natural rubber latex allergy. J Allergy Clin Immunol 2002; 110: S70-74.

54. Hunt LW, Boone-Orke JL, Fransway AF, Fremstad CE, Jones RT, Swanson MC, et al. Amedical-center-wide, multidisciplinary approach 
to the problem of natural rubber latex alergy. J Occup Environ Med 1996; 38: 765-770.

55. Hunt LW, Kelkar P, Reed CE, Yunginger JW. Management of occupational allergy to natural rubber latex in a medical center: the importance of quantitative latex allergen measurement and objective follow-up. JAllergy Clin Immunol 2002; 110: S96-106.

56. Sussman GL, Liss GM, Deal K, Brown S, Cividino M, Siu S, et al. Incidence of latex sensitization among latex glove users. J Allergy Clin Immunol 1998; 101: 171-178.

57. Phillips VL, Goodrich MA, Sullivan TJ. Health care worker disability due to latex allergy and asthma: a cost analysis. Am J Public Health 1999; 89: 1024-1028.

58. Bolyard EA, Tablan OC, Williams WW, Pearson ML, Shapiro CN, Deitchman SD, et al. Guideline for infection control in health care personnel, 1998. AJIC 1998; 26: 289-354.

59. De La Hoz Caballer B. Estrategias para la prevención de la alergia al látex. En: Blanco Coverra C, Quirce Gancedo S (ed). Alergia al látex 2002. Sociedad Española de Alergia e Immunología Clínica. Barcelona: MRAEditions; 2002: 213-244.

60. Allmers H, Brehler R, Chen Z, Rauf-Heimsoth M, Fels H, Baur X. Reduction of latex aeroallergens and latex-specific IgE antibodies in sensitized workers after removal of powered natural rubber latex gloves in a hospital. J Allergy Clin Immunol 1998; 102: 841-846.

61. Charous BL, Schuenemann PJ, Swanson MC. Passive dispersion of latex aeroallergen in a healthcare facility.Ann Allergy Asthma Immunol 2000; 85: 285-290.

62. Swanson MC, Bubak ME, Hunt LW, Yunginger JW,Warner MA, Reed CE. Quantification of occupational latex aeroallergens in a medical center. J Allergy Clin Immunol 1994; 94: 445-451.

63. Quirce S, Swanson MC, Fernandez-Nieto M, de las Heras M, Cuesta J, Sastre J. Quantified environmental challenge with absorbable dusting powder aerosol from natural rubber latex gloves. J Allergy Clin Immunol 2003; 111: 788-794.

64. Cremer R, Hoppe A, Kleine-Diepenbruck U, Blaker F. Effects of prophylaxis on latex sensitization in children with spina bifida. Eur $\mathbf{J}$ Pediatr Surg 1998; 8: 59.

65. Cremer R, Kleine-Diepenbruck U, Hering F, Holschneider AM. Reduction of latex sensitization in spina bifida patients by a primary prophylaxis programme (five years experience). Eur J Pediatr Surg 2002; 12: S19-21

66. Nakamura CT, Ferdman RM, Keens TG, Davidson Ward SL. Latex allergy in children on home mechanical ventilation. Chest 2000; 118 : 1000-1003.

67. Gentili A, Ricci G, Di Lorenzo FP, Pigna A, Masi M, Baroncini S. Latex allergy in children with oesophageal atresia. Paediath Anaesth 2003; 13: 668-675.

68. Kotilainen HR. Latex and vinyl examination gloves. Quality control procedures and implications for health care workers. Arch Intern Med 1989; 149: 2749-2753

69. Crippa M, Belleri L, Mistrello G, Carsana T, Neri G, Alessio L. Prevention of latex allergy among health care workers: evaluation of the extractable latex protein content in different types of medical gloves. Am J Ind Med 2003; 44: 24-31.

70. Ownby DR. Strategies for distinguishing asymptomatic latex sensitization from true occupational allergy or asthma. Ann Allergy Asthma Immunol 2003; 90: S42-46

71. Kotilainen HR, Avato JL, Gantz NM. Latex and vinyl nonsterile examination gloves: status report on laboratory evaluation of defects by physical and biological methods. Appl Environ Microbiol 1990; 56: 16271630.

72. Charous BL, Blanco C, Tarlo S, Hamilton RG, Baur X, Beezhold D, et al. Natural rubber latex allergy after 12 years: recommendations and perspectives. J Allergy Clin Immunol 2002; 109: 31-34.

73. Baur X. I are we closer to developing threshold limit values for allergens in the workplace?. Ann Allergy Asthma Immunol 2003; 90: S1118. 